\title{
Pseudo-Spectrum Time Domain and Time Reversal Mirror technique using in Microwave-induced Thermo-Acoustic Tomography System
}

\author{
Guoping Chen ${ }^{1,2}$, \\ ${ }^{1}$ School of Communication and Information Engineering, Chongqing 400065, China \\ ${ }^{2}$ School of Electronic Engineering, Chengdu 610054, China \\ Email: chengp@cqupt.edu.cn \\ Zhiqin Zhao ${ }^{2}$ and Qing.H. Liü ${ }^{2,3}$ \\ ${ }^{2}$ School of Electronic Engineering, Chengdu 610054, China \\ ${ }^{3}$ Department of Electrical and Computer Engineering, Duke University, Durham, NC 27708, USA \\ Email: zqzhao@uestc.edu.cn
}

\begin{abstract}
Microwave-Induced Thermo-Acoustic Tomograpphy (MITAT) has attracted more concerns in recent years in biomedical imaging field. It has both the high contrast of the microwave imaging and the high resolution of ultrasound imaging. As compared to optoacoustics, which uses instead a pulsed light for evoking optoacoustic response, thermo-acoustic imaging has the advantage of deeper tissue penetration, attaining the potential for wider clinical dissemination, especially for malignant tumors. In this paper, the induced thermo-acoustic wave propagating in a mimic biologic tissue is simulated by numeric method Pseudo-Spectrum Time Domain (PSTD). Due to the excellent performance in noisedepress and the stability for the fluctuation of the model parameters, Time Reversal Mirror (TRM) imaging technique is studied computationally for the simulative received thermo-acoustic signals. Some thermo-acoustic objects with different initial pressure distribution are designed and imaged by TRM technique to represent the complex biologic tissue case in a random media. The quality of images generated by TRM technique based on PSTD method hints the potential of the MITAT technique.
\end{abstract}

Index Terms-Microwave-Induced Thermo-Acoustic Tomography, Pseudo-Spectrum Time Domain, Time Reversal Mirror

\section{INTRODUCTION}

Recent research has suggested that microwave -induced thermo-acoustic tomography can be a powerful imaging technology with good spatial resolution. In a MITAT system, a narrow pulse modulated microwave irradiation is used to illuminate the biologic tissue. When the pulsed microwave irradiation is absorbed by a tissue, the heating and subsequent expansion of the tissue give

Manuscript received January 1, 2008; revised June 1, 2008; accepted July 1, 2008.

Copyright credit, project number, corresponding author, etc. rise to an instantaneous acoustic stress or pressure distribution inside the tissue. The induced pressure distribution prompts acoustic wave propaga- tion toward the surface of the tissue with various time dalays. The ultrasound transducers, which can convert mechanical stress into electrical signals, are placed around the tissue to record these outgoing acoustic waves, commonly referred to as thermo-acoustic signals. These thermo-acoustic signals carry information about the acoustic properties of the tissue. So using these detected acoustic waves can compute the distribution of the initial acoustic pressure or microwave absorption, which is related to the properties of the tissue. Microwave-induced thermo-acoustic tomography in biological tissues combines the advantages of pure microwave imaging and pure ultrasound imaging [1], [2]. As a microwave pulse is used to irradiate the tissue, the image of a MITAT system has higher contrast than a conventional ultrasound system. This is because that the high electrical (relative permittivity and electrical conductivity) contrasts due to the significantly different sodium concentrations, fluid contents and electrochemical properties in the different tissues, especially between the normal soft tissue and malignant tumors (For example, the ratios of relative permittivity and electrical conductivity between the normal soft tissue and malignant tumors are 1:3.75 and 1:6.75 respectively at the frequency of $800 \mathrm{MHz}$ ) [3]. On the other hand, the MITAT image has higher resolution than the conventional microwave imaging. Because the velocity of acoustic wave in the soft tissue is $1.5 \mathrm{~mm} / \mathrm{us}$, thermo-acoustic signals at megahertz can provide millimeter or better spatial resolution (Generally, the irradiated microwave in MITAT system works in the frequency range of $400 \sim 3000 \mathrm{MHz}$, and the corresponding wavelength is in the range of $100 \sim 750 \mathrm{~mm}$, while the received ultrasound is in the frequency range of $200 \sim 2000 \mathrm{KHz}$, and the 
corresponding wavelength is in the range of $0.75 \sim 7.5 \mathrm{~m}$ $\mathrm{m})$. Furthermore, a MITAT system utilizes non-ionizing microwave pulse radiation; therefore it causes less harm to human body compared with the X-ray imaging system [4].

However, due to the heterogeneous of the biologic tissue, the induced thermo-acoustic wave propagating in it is affected. The imaging algorithm, such as BackProjection (BP), neglected this situation will decrease the contrast and resolution of the image. Time Reversal Mirror (TRM) technique [5] is a combination based on wave propagation and array signals process and can well employ the propagation information and achieve much better resolution especially in the complex environments. It has the ability to effectively suppress system noise due to the characteristics of its spatial-time matched filtering. Further more, the statistic stability due to the self-average character of TRM technique makes it has the capability to decrease the effects induced by random fluctuating of the media parameter. So this technique is a good choice for biomedical tomography.

In this paper, we present the Time Reversal Mirror technique based on Pseudo-Spectrum Time Domain method for the microwave-induced thermo-acoustic imaging of biological tissue. Numerical simulations are given to illustrate the validity of our methods.

\section{II .THEORETICAL BACKGROUND}

The thermo-acoustic effect refers to the induction of acoustic radiation following temperature elevation due to absorption of microwave pulse energy. The magn- itude of thermo-acoustic response is proportional to th- e local absorbed power density and thermoelastic properties of the imaged tissue. The thermo-acoustic theory has been discussed in many literature reviews such as [6]. Here, we briefly review only the fundam- ental equations. The pressure $p(\vec{r}, t)$ at position $\vec{r}$ and time $\mathrm{t}$ in an acoustically homogeneous medium in respond to a heat source $H\left(\vec{r}^{\prime}, t^{\prime}\right)$ obeys the following equation [7]:

$$
\nabla^{2} p(\vec{r}, t)-\frac{1}{c^{2}} \frac{\partial^{2}}{\partial t^{2}} p(\vec{r}, t)=-\frac{\beta}{C_{p}} \frac{\partial}{\partial t} H\left(\vec{r}^{\prime}, t^{\prime}\right),
$$

Where $\left(\vec{r}^{\prime}, t^{\prime}\right)$ and $(\vec{r}, t)$ mean that spatial and time coordinates of source and the ultrasound transducer respectively. $H\left(\vec{r}^{\prime}, t^{\prime}\right)$ is the heating function defined as the thermal energy deposited by the energy source per time and volume, $\beta$ is the isobaric volume expansion coefficient, $C_{p}$ is the heat capacity. $c$ is the velocity of ultrasound wave, in most biologic tissue, its value is about $1.5 \mathrm{~mm} / \mathrm{us}$. The heating function $H\left(\vec{r}^{\prime}, t^{\prime}\right)$ can be written as the product of a spatial absorption function and a temporal illumination function of the microwave source:

$$
H\left(\vec{r}^{\prime}, t^{\prime}\right)=I_{0} A\left(\vec{r}^{\prime}\right) \cdot \eta\left(t^{\prime}\right)
$$

Where $I_{0}$ is the amplitude of the microwave pulse, $\eta\left(t^{\prime}\right)$ is the temporal profile of the microwave pulse. $A\left(\vec{r}^{\prime}\right)$ is the spatial distribution of the microwave energy absorbed in the tissues. If the microwave pulse duration is short enough so that both heat and temporal stress are confined to the size of the desired resolutionlimited voxel [8], one may assume the excitation to be a delta function in time

$$
\eta\left(t^{\prime}\right)=\delta\left(t^{\prime}\right)
$$

So, the solution of equation (1) based on the free space Green's function in the time domain can be expressed as:

$$
p(\vec{r})=G\left(\vec{r}, \vec{r}^{\prime}\right) \otimes p_{a}(\vec{r}),
$$

Where $G\left(\vec{r}, \vec{r}^{\prime}\right)$ is Green's function, and the integral volume is a sphere defined by $\left|\vec{r}-\vec{r}^{\prime}\right|=\left|t-t^{\prime}\right| \cdot c, \otimes$ is the convolute operator, and

$$
p_{a}(\vec{r})=\frac{\beta I_{0}}{c_{\rho}} \iiint_{\left|\vec{r}-\vec{r}^{\prime}\right|=\left|t-t^{\prime}\right| c} A\left(\vec{r}^{\prime}\right) d \vec{r},
$$

Where $p_{a}(\vec{r})$ depicts observed pressure induced by the $A\left(\vec{r}^{\prime}\right)$ enclosed in the sphere $\left|\vec{r}-\vec{r}^{\prime}\right|=\left|t-t^{\prime}\right| \cdot c$. Obviously, $p_{a}(\vec{r})$ can be got by initial pressure defined in $\left|\vec{r}-\vec{r}^{\prime}\right| \rightarrow 0$ case:

$$
\begin{aligned}
& p_{a}(\vec{r})=\iiint_{\left|\vec{r}-\vec{r}^{\prime}\right|=\left|t-t^{\prime}\right| c} p_{\delta}\left(\vec{r}^{\prime}\right) d \vec{r} \\
& p_{\delta}\left(\vec{r}^{\prime}\right)=\left.\frac{\beta I_{0}}{c_{\rho}} A\left(\vec{r}^{\prime}\right)\right|_{\vec{r} \rightarrow \vec{r}^{\prime}},
\end{aligned}
$$

The purpose of MITAT is to reconstruct the spatial distribution of the microwave energy absorbed function $A\left(\vec{r}^{\prime}\right)$ based on the received ultrasound pressure wave $p(\vec{r}, t)$, and we can see from the equation (6) that the relationship between the initial pressure $p_{\delta}\left(\vec{r}^{\prime}\right)$ and the microwave energy deposition $A\left(\vec{r}^{\prime}\right)$ distribution is proportional. So in MITAT system, the reconstruction of the distributions of the absorbed microwave energies $A\left(\vec{r}^{\prime}\right)$ in biologic tissue is equivalent to the reconstruction of the distributions of the microwave induced thermo-acoustic sources $p_{\delta}\left(\vec{r}^{\prime}\right)$.

\section{III.TIME REVERSAL MIRROR}

MITAT is an imaging problem for targets in multiple physic field, the noise accompanying with measurement is inevitable. Therefore, the studies of MITAT focus on how to acquire signal with high SNR and on the image algorithms with high stability for random parameter. The TRM technique which combines the process of wave propagating and array signal processing has become a hot point in recent years. It has significant meaning for applying TRM in MITAT.

Here we briefly describe the theory of TRM.TRM principle is that the received signals are re-emitted into the media with time reversal sequence, and refocused at 
the source location. The ultrasonic wave propagating is called forward procedures, while the re-emitting is called backward procedures.

So there have two processes in a time reversal procedure includes the source propagates in the media, and the received signals are re-emitted into the same media. The first process can be depicted by equation (4), the re-emitting and refocus imaging process can be depicted by a convolution between the $p(\vec{r})$ and Green's function again

$$
p^{T}(\vec{r})=G\left(\vec{r}, \vec{r}^{\prime}\right) \otimes p_{\delta}\left(\vec{r}^{\prime}\right) \otimes G\left(\vec{r}^{\prime}, \vec{r}\right),
$$

For the spatial reciprocity of the Green's function, equation (7) can be rewritten as

$$
\begin{aligned}
& P^{T}(\vec{r})=p_{\delta}\left(\vec{r}^{\prime}\right) \otimes \Gamma\left(\vec{r}, \vec{r}^{\prime}\right) \\
& \Gamma\left(\vec{r}, \vec{r}^{\prime}\right)=G\left(\vec{r}, \vec{r}^{\prime}\right) \otimes G\left(\vec{r}^{\prime}, \vec{r}\right)
\end{aligned}
$$

Where $\Gamma\left(\vec{r}, \vec{r}^{\prime}\right)$ means the system function of the TRM process.

\section{A. Spatial-temporal filtering of TRM}

Generally, the measured signals have noise in an actual MITAT system, so the received signals in forward procedure should be modified as

$$
p_{\delta}^{\prime}\left(\vec{r}^{\prime}\right)=p_{\delta}\left(\vec{r}^{\prime}\right)+n,
$$

Where $n$ is an additive noise function, equation (8) should be modified in terms of the received signals model defined in equation (9),

$$
\begin{aligned}
& p^{T}\left(\vec{r}^{\prime}\right)=p_{\delta}^{\prime}\left(\vec{r}^{\prime}\right) \otimes \Gamma\left(\vec{r}, \vec{r}^{\prime}\right) \\
& =p_{\delta}\left(\vec{r}^{\prime}\right) \otimes R\left(\vec{r}, \vec{r}^{\prime}\right)+n \otimes G\left(\vec{r}, \vec{r}^{\prime}\right),
\end{aligned}
$$

Where $R\left(\vec{r}, \vec{r}^{\prime}\right)$ is the self correlation function of $\Gamma\left(\vec{r}, \vec{r}^{\prime}\right)$. For the ultrasonic wave $p_{\delta}\left(\vec{r}^{\prime}\right)$ propagating through the system defined by $G\left(x_{f}, x_{p}\right)$ function and the received signals propagating again this system in reversal time sequence, the refocused point is enhanced $N$ times due to the in-phase accumulation by time reversal procedure, while the noise has not this character contrarily. In fact, the maximum of the refocu- sed function $p^{T}\left(\vec{r}^{\prime}\right)$ can be got at $\vec{r}=\vec{r}^{\prime}$ due to the function $R\left(\vec{r}, \vec{r}^{\prime}\right)$. This character of the TRM technique is usually called spatial-temporal filtering.

\section{B. Self-average character of TRM}

Self-average is another character of TRM, which means that time reversal procedure has good statistical stability for a heterogeneous media. Given the maximu$\mathrm{m}$ of the ultrasonic wavelength is far less than the distance between source and the receiver, self-average is determined by the independent of the function $\widehat{\Gamma}\left(\vec{r}, \vec{r}^{\prime}, \omega\right)$ for different frequency $\omega_{1}, \omega_{2}$ [9]:

$$
\begin{aligned}
& E\left\{\hat{\Gamma}\left(\vec{r}, \vec{r}^{\prime}, \omega_{1}\right) \cdot \hat{\Gamma}\left(\vec{r}, \vec{r}^{\prime}, \omega_{2}\right)\right\} \approx \\
& E\left\{\hat{\Gamma}\left(\vec{r}, \vec{r}^{\prime}, \omega_{1}\right)\right\} \cdot E\left\{\hat{\Gamma}\left(\vec{r}, \vec{r}^{\prime}, \omega_{2}\right)\right\} \quad \omega_{1} \neq \omega_{2}
\end{aligned},
$$

Where $E\{\cdot\}$ is the operator of expectation. And there has,

$$
\begin{aligned}
& E\left\{\Gamma\left(\vec{r}, \vec{r}^{\prime}, t\right)^{2}\right\}= \\
& E\left\{\int_{-\infty}^{+\infty} d \omega_{1} \int_{-\infty}^{+\infty} d \omega_{2} e^{-i\left(\omega_{1}+\omega_{2}\right) t} \widehat{\Gamma}\left(\vec{r}, \vec{r}^{\prime}, \omega_{1}\right) \cdot \widehat{\Gamma}\left(\vec{r}, \vec{r}^{\prime}, \omega_{2}\right)\right\}, \\
& \approx \int_{-\infty}^{+\infty} d \omega_{1} \int_{-\infty}^{+\infty} d \omega_{2} e^{-i\left(\omega_{1}+\omega_{2}\right) t} E\left\{\widehat{\Gamma}\left(\vec{r}, \vec{r}^{\prime}, \omega_{1}\right)\right\} . \\
& E\left\{\widehat{\Gamma}\left(\vec{r}, \vec{r}^{\prime}, \omega_{2}\right)\right\}=E^{2}\left\{\Gamma\left(\vec{r}, \vec{r}^{\prime}, t\right)\right\}
\end{aligned}
$$

For an arbitrary small plus value $\gamma>0$, the relationship between the function $\Gamma\left(\bar{r}, \bar{r}^{\prime}, t\right)$ and its expectation can be deduced by Chebyshev's inequality:

$$
\begin{aligned}
& \operatorname{Prob}\left\{\left|\Gamma\left(\vec{r}, \vec{r}^{\prime}, t\right)-E\left\{\Gamma\left(\vec{r}, \vec{r}^{\prime}, t\right)\right\}\right|>\gamma\right\} \leq \\
& \frac{1}{\gamma^{2}} E\left\{\left[\Gamma\left(\vec{r}, \vec{r}^{\prime}, t\right)-E\left\{\Gamma\left(\vec{r}, \vec{r}^{\prime}, t\right)\right\}\right]^{2}\right\} \approx 0
\end{aligned},
$$

The equation (12) depicts that there has the time and frequency domain equivalent relationship for the expectation of function $\Gamma$. And $\Gamma\left(\vec{r}, \vec{r}^{\prime}, t\right) \approx E\left\{\Gamma\left(\vec{r}, \vec{r}^{\prime}, t\right)\right\}$ can be deduced by the equation (13), that is, $\Gamma\left(\vec{r}, \vec{r}^{\prime}, t\right)$ has the self-average character in statistics.

\section{Pseudo-Spectrum Time Domain Method}

The core of the TRM is the system function $\Gamma\left(\vec{r}, \vec{r}^{\prime}, t\right)$. The exact Green's function is difficult to present by analytical method in terms of the complexity in an actual media. However the numerical method is feasible for its flexibility in modeling and computer efficiency. In this paper, the numeric method Pseudo-Spectrum Time Domain (PSTD) is employed to complete the forward core in a TRM procedure, and the PSTD can also numerical presents the Green's function of the complex biologic tissue easily [10], [11]. The Pseudo-S pectrum time domain method is a higher order approxi- mation method in the whole domain which can use the FFT to calculate the spatial derivatives.

For the finite difference time domain (FDTD) method uses one order partial differential equation, the equation (1) should be rewritten as:

$$
\begin{aligned}
& \frac{\partial \vec{v}}{\partial t}=-\frac{1}{\rho} \nabla p \\
& \frac{\partial p}{\partial t}=-K \nabla \cdot \vec{v}
\end{aligned}
$$

Where $K=\rho c^{2}, \vec{v}$ is the velocity of elastic media cub defined in Fig. 1, which equals to $\partial u_{i} / \partial t$. The numuerical system function can get by discretizing equatio- $\mathrm{n}$ (14) into $N=N x \times N y(N x, N y$ are total nodes along $x$ and $y$ directions, respectively) orthogonal coordinates. 


$$
\begin{aligned}
& \frac{\partial W}{\partial t}=f(W), W \equiv\left(v_{x}, v_{y}, p\right)^{T} \\
& f(W)=\left(-\frac{1}{\rho} \frac{\partial p}{\partial x},-\frac{1}{\rho} \frac{\partial p}{\partial y},-K\left(\frac{\partial v_{x}}{\partial x}+\frac{\partial v_{y}}{\partial y}\right)\right)^{T}
\end{aligned}
$$

Where superscript ' $T$ ' means matrix transpose, $v_{x}, v_{y}$ are the velocity components of $\vec{v}$ along $x, y$ directions.

For the one order partial differential equation regardless of $v_{x}, v_{y}, p$ or $W$ in time domain , equation (15) can be calculated by corresponding form in the frequency domain, and this transform can be implemented by Fast Fourier Transforms (FFT) and Inverse Fast Fourier Transforms (IFFT) algorithm effectively. The Pseudo-Spectrum Time Domain (PSTD) method is torched under those ideals. For its global high order characteristics, far less nodes are needed than primitive FDTD method (in order to obtain numeric precision, usually 12-20 nodes are needed in primitive FDTD, while only 2-8 nodes are needed in the PSTD). So, the computation scale can be enlarged and the efficiency can be advanced.

So the basic steps of PSTD are summarized as follows:

1)Using FFT,generate $\bar{u}_{0}, \bar{u}_{1}, \bar{u}_{2}, \ldots \bar{u}_{N-1}$

for the nodes discretized from eq.(14)

2)Calculating $j k \bar{u}(k)$ by multiplying

$\bar{u}_{0}, \bar{u}_{1}, \bar{u}_{2}, \ldots \bar{u}_{N-1}$ by the discretized spa

-tial frequencies,i.e.,

3)Obtaining $\frac{d}{d x} u(x)$ by using the IFFT

of $j k \bar{u}(k)$

\section{V.COMPUTER SIMULATION}

To validate the availability of TRM technique based on PSTD method, a simplified model with two layers structures immersed into mineral oil is used to mimic the biologic tissue background. The out layer is a 3mm thick skin, the inner is fat tissue, their density and ultrasonic velocity parameter details are defined by literature [12]. The scales of simulative domain are $76 \mathrm{~mm} \times 76 \mathrm{~mm}$, the total discrete nodes are $N=512 \times 512$, in which 8 nodes Perfectly Matched Layer (PML) are assigned at each edge around. The time step is set as $1.860 \mathrm{e}-2 \mu \mathrm{s}$, and the total iterative steps are 2900. 128 sensors are equably positioned at a circle with a radius $35 \mathrm{~mm}$. In the forward procedure, the thermo-acoustic source is set as Gaussian pulse with the center frequency $1.5 \mathrm{MHz}$ 、 relative bandwidth $B W=1.0$, duration $1.35 \mu \mathrm{s}$ and maximum pressure of $10^{6}$ Pascal (initial pressure of other simulative domain is set at $10^{5}$ Pascal), the thermo-acoustic waves are propagating and received by the 128 sensors. While in the backward procedure, the received signals are re-emitted into the simulative domain by these sensors in time reversal sequence, and refocused imaging. For the computation- al scale is uniform, each simulative procedure spends about 20 minutes alike to get an image using Matlab codes at a desktop computer with AMD5000+ CPU, 2G memory configurations.

Four objects are located in this simplified two layers background model, two circles and two rectangles constructed like a 'face', with different densities and velocities to the inner fat tissue, is used to simulate the shapes of various tumors. And the each object, with different initial pressure, is used to represent the thermo-acoustic sources generated by various tumors in actual tissue.

\section{A. Image generated by TRM for different initial pressu- re objects in a certain parameter model}

Firstly, the capacity of the TRM in terms of the four objects with different initial pressure in a certain ultrasonic velocity parameter model is studied. The objects and the background velocity distributions are depicted in Fig.1. The four objects are designed with 1.0, $0.5,0.25,0.125,0.1$ time strength of the initial pressure value. Fig. 2 and Fig. 3 are the image generat- ed by BP and TRM technique.

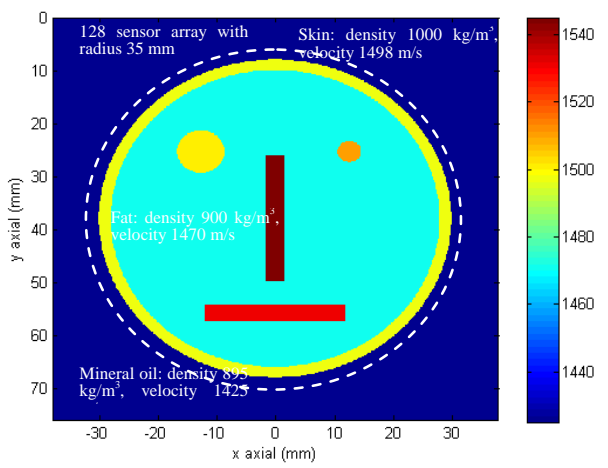

Figure.1: The certain parameter model (total nodes are: 512 x 512)

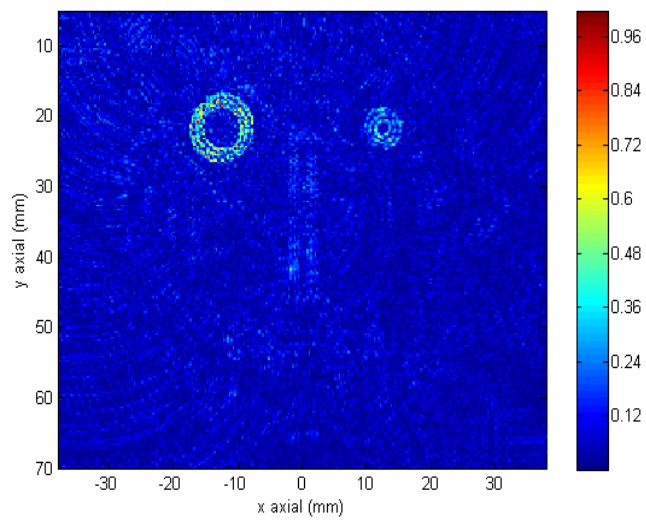

Figure 2. Image generated by BP 


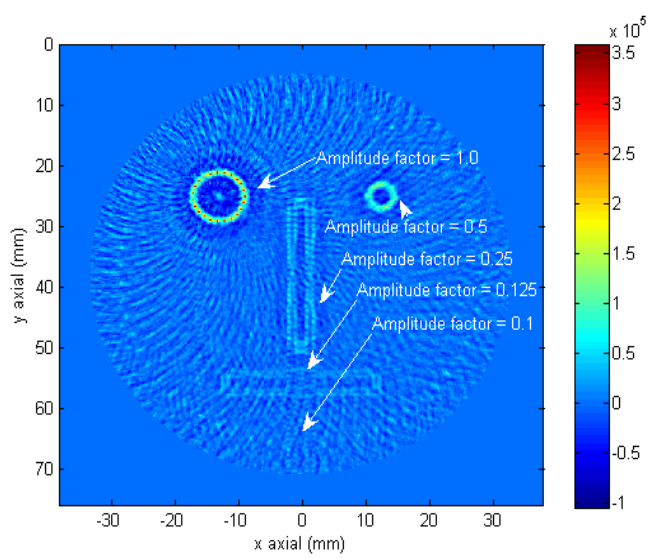

Figure 3. Image generated by TRM.

It can be read from the Fig.2 and Fig.3 that images generated by $\mathrm{BP}$ and TRM technique are both can distinguish the four objects. Both images are refocused very well at the original initial pressure sources as expected. The different area has been clearly imaged with different strength also. The weak objects such as the mouth in the image generated by BP method can read reluctantly, while the mouth imaged by TRM technique can read clearly.

$B$. The image generated by TRM for the received signal$s$ with added noise

Secondly, a more close to actual situation that the received signals are contaminated by noise is studied. In this case, given the model and objects still have certain velocity parameter distributions, but the re-emitted signals are the received signals with added Gaussian White noise artificially. Fig. 4 is the compare between the original signal and the contaminated signals by add- ed noise. The average amplitude comparison between the original signal and the contaminated signals is $-10 \mathrm{~dB}$. Fig. 5 and Fig. 6 are the images generated by BP and TRM technique with contaminated signals.

Despite promising characteristics, MITAT has not yet become a mainstream imaging approach partly due to signal-to-noise ratio(SNR). With the character of spatial-temporal matched filtering and self-average, TRM

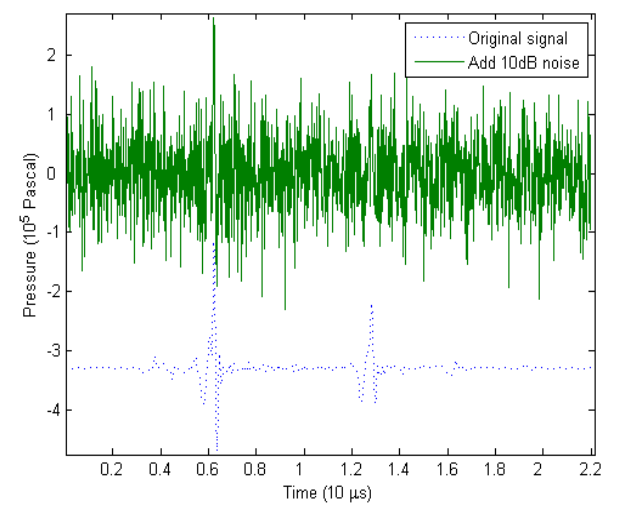

Figure 4. The compare between the original and the added noise

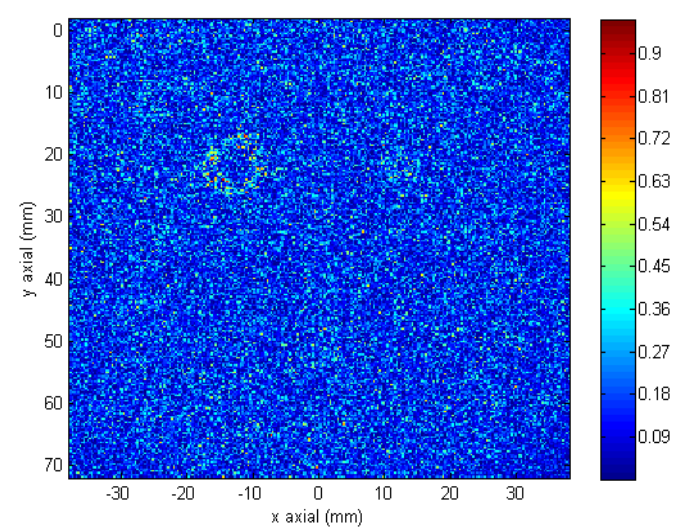

Figure 5. Image generated by BP with contaminated signals

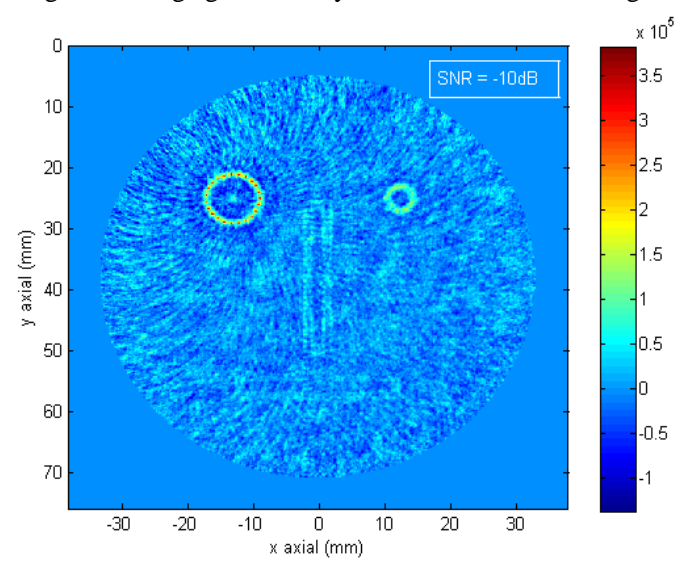

Figure 6. Image generated by TRM with contaminated signals.

can well improve the problem of SNR.

The refocused image generated by TRM is agreed very well with the objects defined in Fig. 4 with good contrast. The weakest strength of the focused image mouth is larger than the averaged background $20 \mathrm{~dB}$. While the image generated by $\mathrm{BP}$ algorithm is low-grade even in contrast and resolution, only big eye with the strongest initial pressure in all the four objects can be distinguished actually, and the contrast of it is about $3 \mathrm{~dB}$ to the average background value.

\section{The self-average character of the TRM technique in a random parameter model}

At last, the self-average capacity of the TRM technique for a model with random velocity parameter is studied in terms of the heterogeneousness of an actual tissue. Fig.7 is the map of the random velocity parameter distribution of the model and the objects. The random amplitude ratio to the certain model is $10 \%$. Fig. 8 is the image generated by TRM technique in this random model with $\mathrm{SNR}=-10 \mathrm{~dB}$ added noise signals same as the $\mathrm{V}$. B schedule. 


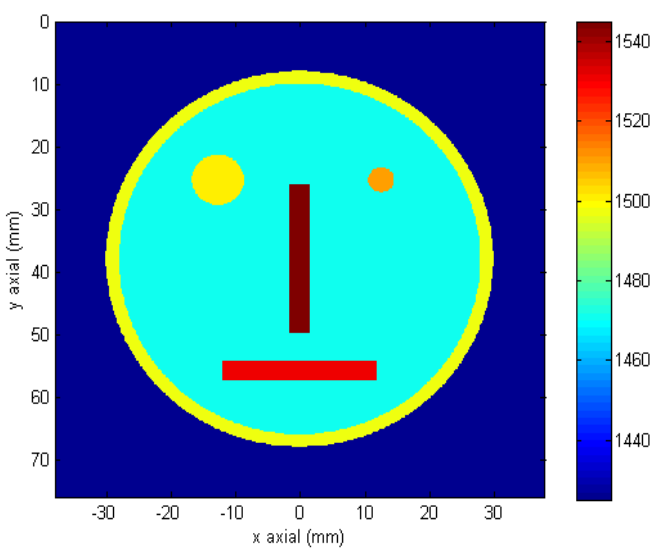

Figure 7. The random model

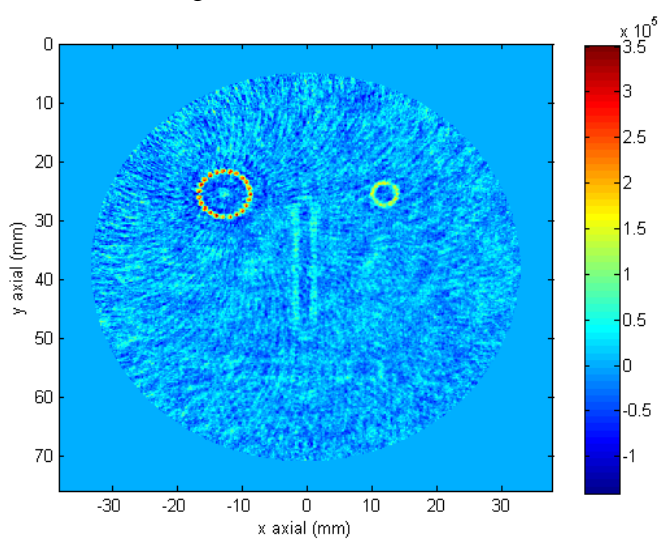

Figure 8. Image generated by TRM in random model

From Fig. 8, we can clearly see that the refocused image is agreed very well with the objects defined in Fig. 1 all the same. The contrast of the image is still very well. The weakest strength of the focused image mouth is larger than the averaged background $18 \mathrm{~dB}$.

Three schedulers are designed to validate the capacities of the TRM technique depicted in the section III, and the images generated by TRM technique based on PSTD presents good contrast and high resolution no matter what the strong noise and the model with big random parameter cases.

\section{CONCLUSIONS}

In this paper, the proportional relationship between the microwave energy deposition and the induced thermo-acoustic source distribution is derived. As the time reversal mirror (TRM) technique has great potential in noise suppression and self-average character for the model with random parameter fluctuation, the TRM technique based on the Pseudo-Spectral Time Domain (PSTD) method is introduced in MITAT system. In order to validate the performance of TRM in the imaging of MITAT, some two-dimensional simulative images are studied and compared with the BP algorithm. It has been shown that the images generated by TRM technique have very good performance in contrast and resolution for different initial pressure objects, this hints the potential of the MITAT technique.

\section{ACKNOWLEDGMENT}

This paper is supported by China NSF (No. 60927002), China Postdoctoral Science Foundation (No. 20100470077), Chongqing Education Commission program (No.KJ100520), Supported by Natural Science Foundation Project of CQ CSTC (CSTC, 2010BB2419).

\section{REFERENCES}

[1]. A. Kruger, W. L. Kiser, D.R. Reinecke, G.A. Kruger, R.L. Eisenhart, Thermoacoustic Computed Tomography of the Breast at $434 \mathrm{MHz}$, IEEE MIT-S Digest, vol. 2, pp. 591 595, 1999.

[2]. M. Xu, L. V. Wang, RF-induced Thermo-acoutic Tomography, Proceedings of the Second Joint EMBS/BMES Conference, pp. 1211 - 1212, 2002.

[3]. W. T. Joines, Y. Zhang, C. Li, R. L. Jirtle, The measured electrical properties of normal and malignant human tissues from 50 to $900 \mathrm{MHz}$, Am.Assoc. Phys. Med., vol. 21, pp. 547-551, 1994.

[4]. K. H. Lim, J. H. Lee, Q. H. Liu, Thermoacoustic tomography forward modeling with the spectral element method, Medical Physics, 2008, 35(1): 4-12.

[5]. M. Fink, Time Reversal of Ultrasonic Fields. I. Basic principles, IEEE Transaction on Ultrasonics, Ferroelectrics, and Frequency Control, vol. 39, no.5, 555 - 567, 1992.

[6]. A.C.Tam,"Application of photoacoustic sensing techniques,"' Rev.Mod.Phys.,vol.58,pp.381-431,1986

[7]. CHEN GuoPing, ZHAO ZhiQin, GONG Wei, NIE ZaiPing and LIU Qing-Huo, The Development of the Microwave-Induced Thermo-acoustic Tomography Prototype System, Chinese Science Bulletin, 2009, 52(12):1786-1789.

[8]. L.V.Wang,Photoacoustic Imaging and Spectroscopy (CRC,Boca Raton,2009)

[9]. J. P. Fouque, G. Papanicolaou, Wave Propagation and Time Reversal in Randomly Layered Media, Springer Press, 2007.

[10].Q. H. Liu, The PSTD algorithm: A time-domain method combining the pseudospectral technique and perfectly matched layers, J. Acous. Soc. Am., vol. 101, no. 5, Pt. 2, p. 3182, May 1997 (13rd Acoustical Society of America Meeting).

[11].G. Wojcik, Fomberg, B. Waag, R. Carcione, L. Mould, J. Nikodym, L. Driscoll, T. , Pseudo-spectral Methods for Large-Scale Bioacoustic Models, IEEE Ultrasonics Symposium, vol.2, pp. 1501- 1507, Oct. 1997.

[12].T. D. Mast, Empirical relationships between acoustic parameters in human soft tissues, Acoustics Research Letters Online, vol. 37, no. 1, pp. 37-43, 2000.

[13]. Minghua Xu, Lihong V.Wang, Time-Domain Reconstruction for Thermoacoustic Tomography in a Spherial Geometry, IEEE Transaction on Medical Imaging, VOL.21,No.7,July 2002

[14].Minghua $\mathrm{Xu}$, Yuan $\mathrm{Xu}$, and Lihong V.Wang, Time-Domain Reconstruction Algorithms and Numerical Simulations for Thermoacoustic Tomography in Various Geometries,IEEE Transactions on Biomedical Engineering,VOL.50,No.9,Sep 2003 


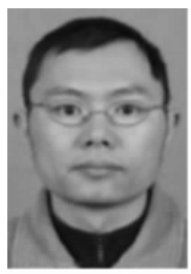

Guoping Chen received M.S. degrees in school of electronic information from the University of Sichuan, Sichuan, China, and the Ph.D. degree in School of Electronic Engineering, University of Electronic Science and Technology of China, in 2006 and 2009, respectively.

In 2009 , he became associate professor with the School of Communication and Information Engineering, Chongqing University of Posts and Telecommunications. His current research interests is Critical technologies of the microwave-induced thermo-acoustic tomography system.

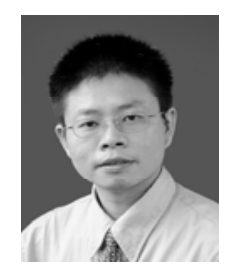

Zhiqin Zhao (SM'05) received the B.S. and M.S. degrees in electronic engineering from the University of Electronic Science and Technology of China (UESTC), Sichuan, China, and the Ph.D. degree in electrical engineering from Oklahoma State University, Stillwater, in 1990, 1993, and 2002, respectively.

From 1996 to 1999, he was with the Department of Electronic Engineering, UESTC. From 2000 to 2002, he researched rough surface scattering as a Research Assistant with the School of Electrical and Computer Engineering, Oklahoma State University. From 2003, he was a Research Associate with the Department of Electrical and Computer Engineering, Duke University, Durham, NC. In 2006, he became a Full Professor with the School of Electronic Engineering, UESTC. His current research interests include computational electromagnetics and signal processing.

Dr. Zhao is a member of Phi Kappa Phi honor society and a senior member of IEEE.

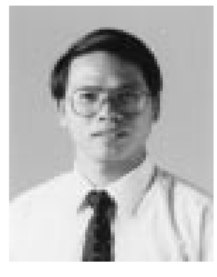

Qinghuo Liu (S'88\{M'89\{SM'94) received the B.S. and M.S. degrees in physics from Xiamen University, China, in 1983 and 1986, respectively,and the Ph.D. degree in electrical engineering from the University of Illinois at Urbana-Champaign in 1989.

From September 1986 to December 1988, Dr. Liu was a research assistant in the Electromagnetics Laboratory at the University of Illinois at Urbana-Champaign. From January 1989 to February 1990 he was a postdoctoral research associate at the same laboratory.From 1990 to 1995 he was a research scientist and program leader with Schlumberger-Doll Research, Ridgefield, CT. Since October 1995 he has been an associate professor at the Klipsch School of Electrical and Computer Engineering, New Mexico State University,Las Cruces, New Mexico.

Dr. Liu is a member of Phi Kappa Phi, Tau Beta Pi, SEG, a full member of U.S. National Committee of URSI Commisions B and F, and a Senior Member of IEEE. Currently he serves as an Associate Editor for IEEE Transactions on Geoscience and Remote Sensing. He received a Presidential Early Career Award for Scientists and Engineers (PECASE) from the National Science and Technology Council (NSTC) and an Early Career Research Award from the Environmental Protection Agency in 1996, and a CAREER Award from the National Science Foundation in 1997. 